\title{
Management of the Midface During Facial Rejuvenation
}

\author{
Andrew P. Trussler, M.D., ${ }^{1}$ and H. Steve Byrd, M.D. ${ }^{1}$
}

\section{ABSTRACT}

The endoscopic midface lift procedure has evolved from experience with postreduction soft tissue repair after facial fracture fixation. The procedure elevates and repositions midface soft tissue, which descends with facial aging; as well, it can correct periorbital congenital abnormalities, such as exorbitism and lateral canthal displacement. The procedure has been refined by the senior author to employ a temporal endoscopic approach alleviating the need for a lower eyelid incision. The plane is sub-SMAS (superficial muscular aponeurotic system) within the pre-zygomatic space with release of the zygo-orbicular ligament and the malar retaining ligament. Using an endoscopically placed suture in the malar retaining ligament, the midface and orbicularis oculi are elevated en bloc, with additional selective sutures applied for specific lower eyelid and cheek morphology. Ancillary lower eyelid procedures including skin resurfacing, skin excision, soft tissue augmentation, and a transblepharoplasty septal reset can all be safely applied to the lower eyelid in the same operative setting. All procedures are technically advanced though once executed deliver an exact correction of the midface, which can be combined with both brow and lower face rejuvenation. The procedure offers limited recovery time and few complications as the facial surgeon becomes facile with the technique.

KEYWORDS: Endoscopic midface lift, midface rejuvenation, lower eyelid rejuvenation

The concepts and principles for the endoscopic midface procedure currently used began in the 1980s when plate fixation for zygomatic fractures was introduced. These plates necessitated wide subperiosteal stripping for exposure and fixation. Postoperatively, patients developed significant soft tissue ptosis that was ascribed to this extensive dissection without adequate soft tissue suspension. Because these plates were to be removed at a second stage, resuspension of the soft tissues through a subperiosteal dissection was subsequently performed at the time of plate removal. To our surprise, we failed to restore the preoperative aesthetics through this approach. In some instances, there was excess bunching and fullness in the lower lid necessitating removal of substantial amounts of lower lid skin and orbicularis oculi muscle that ultimately resulted in very unpredictable lower lid outcomes. Suffice it to say that the final outcome on the operated side seldom succeeded in achieving aesthetic symmetry with the normal unoperated side.

These disappointing results prompted a fresh cadaver anatomic study of a supraperiosteal approach specifically aimed at remaining above the zygomaticus major muscle and below the orbicularis oculi muscle. This approach had the advantage of mobilizing the orbicularis oculi muscle, malar fat pad, and high superficial muscular aponeurotic system (SMAS) as a composite unit while avoiding the downward force of the zygomaticus major muscle by leaving its origin attached
${ }^{1}$ Department of Plastic Surgery, University of Texas Southwestern Medical Center, Dallas, Texas.

Address for correspondence and reprint requests: Andrew P. Trussler, M.D., Assistant Professor, Department of Plastic Surgery, University of Texas Southwestern Medical Center, 1801 Inwood Road, WA4.238, Dallas, TX 75390 (e-mail: andrew.trussler@ utsouthwestern.edu).
Contemporary Face-lift Techniques; Guest Editor, Larry H. Hollier, Jr., M.D., F.A.C.S.

Semin Plast Surg 2009;23:274-282. Copyright (C) 2009 by Thieme Medical Publishers, Inc., 333 Seventh Avenue, New York, NY 10001, USA. Tel: +1(212) 584-4662.

DOI 10.1055/s-0029-1242182. ISSN 1535-2188. 
to the bone. This anatomic approach was adopted as an aesthetic procedure reported in the 1990s as an extended temporal lift. ${ }^{1}$ It reached its full aesthetic value as an endoscopic procedure in the late 1990s where motor innervation to the lower eyelid was further defined and precise suture fixation of the orbicularis muscle identified in a manner that allowed correction of most lower lid problems. ${ }^{2,3}$ However, additional lower eyelid procedures that complement the midface procedures must maintain the orbicularis continuity to preserve the composite midface soft tissue integrity and orbicularis innervation. These have evolved from simple skin excision to transconjunctival fat repositioning, laser skin resurfacing, and micro-fat injection.

\section{EVALUATION}

The overt signs of facial aging are noted in most patients around the age of 40 and are generally periocular in location. The cause is the combined effect of gravity and the activity of the orbicularis oculi muscle. Patients most frequently complain that they look tired in spite of being well rested. In some patients, and certainly among older patient groups, attention is focused on jowl, jawline, and neck laxity. If balanced facial rejuvenation is to be achieved, care must be taken not to overlook the concomitant changes of the upper and mid face in these patients and the value of avertical high SMAS movement when the lower face is addressed. Key points to the evaluation are as follows:

- The component of lateral brow descent, which contributes to the appearance of fatigue and excess weight to the upper eyelid. This lateral excess can camouflage lateral canthal malposition and excess lateral eyelid skin. This upper lid fullness can be corrected at least in part by brow repositioning.

- Malar fat pad descent, which can reveal stigmata of midface aging including a prominent tear trough and a negative vector orbit.

- A widened malar-orbicular crescent, which represents a ptotic lower border of the orbicularis.

- A deepened tear trough and malar groove.

- An accentuated lid/cheek junction, which is the main goal of the procedure in that this definition should be blended and narrowed.

- Improved aesthetics with two-point elevation of the lateral brow and malar complex gives the examiner an estimation of the corrective contribution of the procedure and need for ancillary procedures (Figs. 1 and 2).

\section{ANATOMY}

A key goal in the forehead and midface portion of this procedure is the mobilization and release of the orbicu-

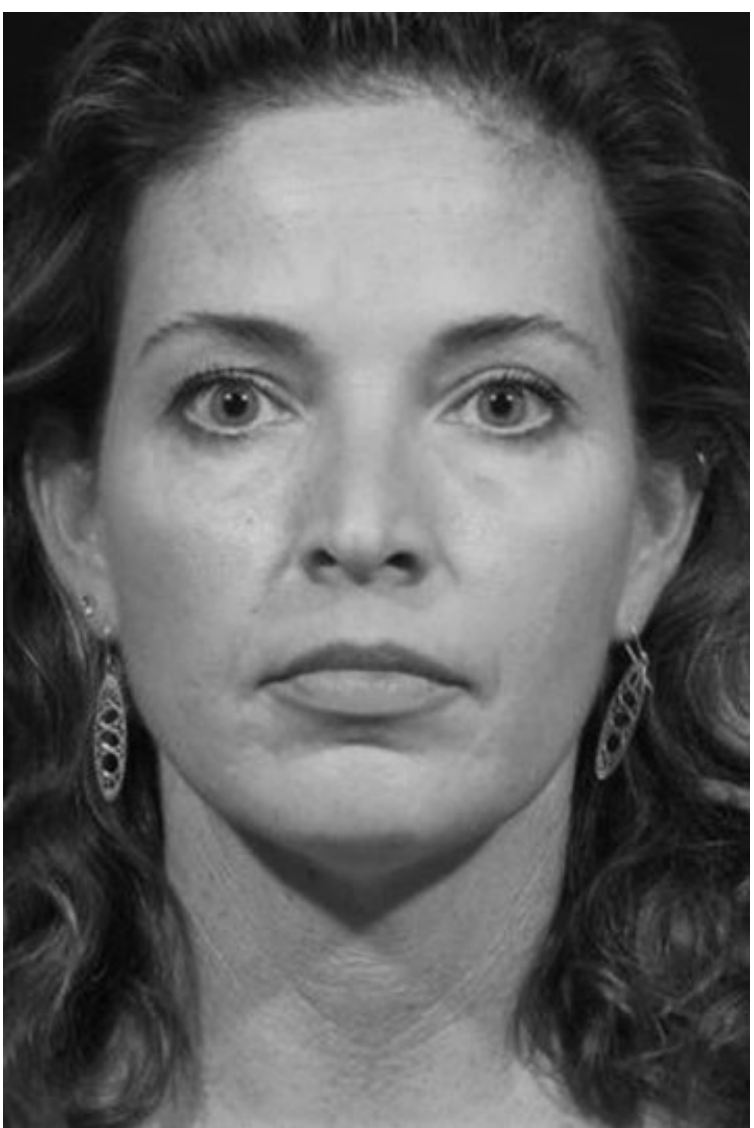

Figure 1 Preoperative patient photograph demonstrating the typical upper and mid face changes including lateral brow descent, upper lid fullness corrected at least in part by brow repositioning, malar fat pad descent, widened malar-orbicular crescent, deepened tear trough and malar groove, and an accentuated lid/cheek junction.

laris oculi muscle from the orbit. The release is above rather than below the periosteum. We believe this obtains more shaping of the brow and an effective shift of the preseptal upper lid orbicularis, which eliminates the need for upper eyelid muscle resection. Critical to the mobility of the orbicularis oculi muscle is the release of the preseptal muscle from its attachments to the orbital rim. In the lower lid, this attachment has been identified as the zygo-orbicular ligament. ${ }^{3}$ Laterally, this same attachment along the outer rim surface represents the superficial head of the lateral canthus, while the upper lid fusion between the orbit and orbicularis is anatomically similar to that described in the lower eyelid. This zone of fusion serves as a retaining structure to the brow. ${ }^{4}$ The selective release, mobilization, and fixation of these retaining structures of the orbicularis oculi muscle are key in achieving the desired periorbital aesthetics. Precise suture placement in the four target zones of the orbicularis oculi muscle allows lower lid shaping and control based on the requirements of the lid morphology (Fig. 3). 


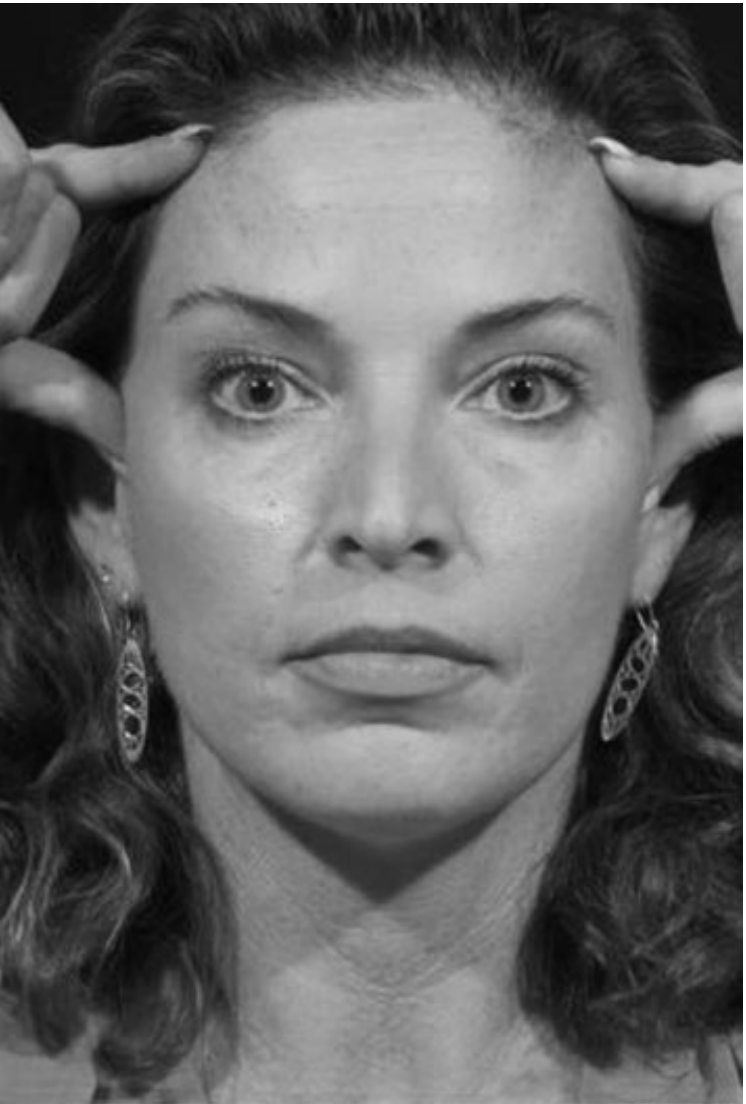

Figure 2 The same patient as shown in Fig. 1 with twofinger elevation of the brow and cheek to mimic correction and demonstrate the improved aesthetics.

A second key in achieving the desired aesthetic relationship between the midface and lower eyelid depends on the release and mobilization of the malar retaining ligament. ${ }^{5}$ This ligament consists of a dimesized zone of fibrous attachments extending from the

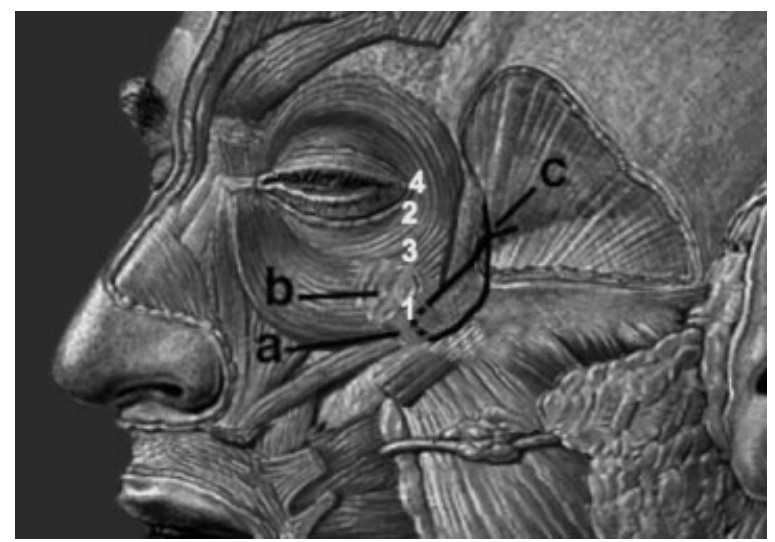

Figure 3 Midface points of suture fixation. Point 1: Malar retaining ligament suture used always for midface elevation. Point 2: Preseptal orbicularis oculi used for scleral show and lid laxity. Point 3: Preorbital orbicularis oculi used for exorbitism. Point 4: Deep head of lateral canthal tendon used for canthal malposition.

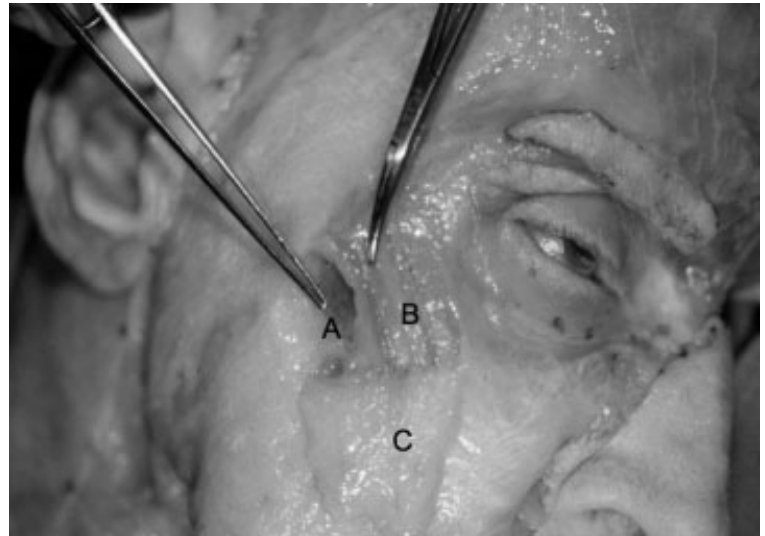

Figure 4 Malar retaining ligament: $A$, zygomaticus major; B, orbicularis oculi; C, malar fat pad (turned down).

periosteum of the zygomatic eminence to the subcutaneous tissue of the cheek. The lateral and most dense portion of this ligament arises from the lateral border of the zygoma where the master muscle attaches. Fibers continue through the origin of the zygomaticus major muscle and extend superficially through the malar fat pad and lateral border of the orbicularis oculi muscle (Fig. 4). By releasing these fibers above the zygomaticus major muscle, the midface can be moved vertically, eliminating the downward force of the zygomaticus muscle that is typically produced in a subperiosteal approach. The anatomic components of this midface movement include the orbital portion of the orbicularis oculi muscle, the malar fat pad, and the upper or high SMAS.

The third anatomic key of the eyelid midface procedure is the sparing of the orbicularis oculi muscle. This is achieved by avoiding: muscle division, muscle resection, and muscle denervation. The motor innervation to the lower lid orbicularis not only arises medially from the zygomatic branch running deep to the zygomaticus major muscle but also laterally from branches arising from the zygomatic branch before it passes

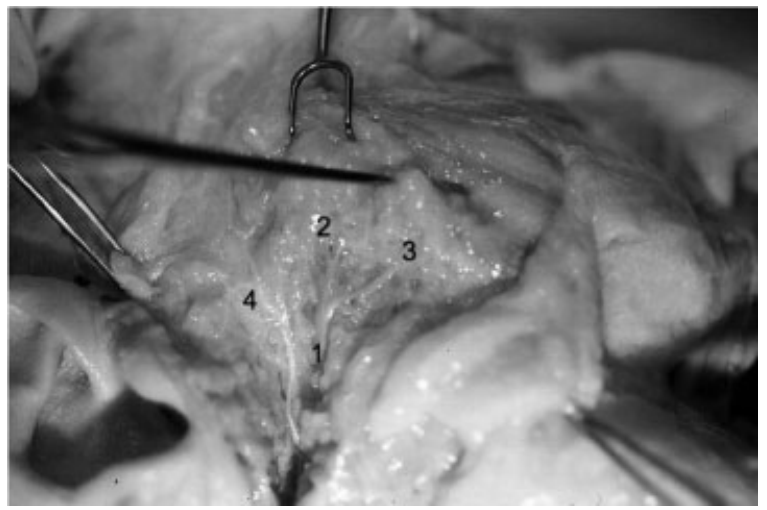

Figure 5 Zygomatic branch of facial nerve: 1, proximal zygomatic branch; 2, lateral division with branches to orbicularis oculi; 3 , medial division traveling under zygomaticus major; 4, frontal branch. 
beneath the zygomaticus major muscle ${ }^{6}$ (Fig. 5). These lateral branches enter the inferior lateral belly of the orbicularis muscle. Although the medial innervation is key to blink, the lateral innervation is equally important to lower lid pretarsal muscle tone. It is for this reason that transpalpebral division of the orbicularis muscle is avoided and a transconjunctival approach is used when access to fat or the septum is needed.

The relevant anatomy to lower facial rejuvenation hinges on the mobilization and fixation of the structures previously described. The important fact to be recognized is that the upper or high SMAS movement has already occurred. Hence, when dealing with the lower face, a skin-only or low SMAS skin procedure is indicated. The vector of the low SMAS or skin procedure is lateral along the jawline. Emphasizing a strong jawline vector while avoiding the additional lateral tension on the high SMAS avoids the tightening and the flattening of the malar remnants with the creation of submalar traction lines that have been referred to as "lateral sweep." In the majority of patients who have significant lower facial laxity, the senior authors' preference is a separate skin and SMAS flap procedure. The SMAS is elevated in continuity with the platysmal muscle so that a strong lateral vector SMAS-platysmal pull is achieved along the jawline. The skin is closed with minimal preauricular tension. The lateral vector SMAS platysma movement generally fails to provide adequate tension in the submental area from the hyoid to the mandible. A medial plication in this zone is generally incorporated. Platysma muscle division is reserved for banding and for very obtuse necks.

\section{TECHNICAL STEPS}

\section{Markings and Incisions}

Markings for the forehead include a vertical brow vector placed at the junction of the medial two thirds and lateral one third of the brow. A point for frontalis muscle fixation is marked along this vector where the vertical forehead begins to slope cephalad. The vector for malar and midface lift is marked across the zygomatic eminence and extends from the nasal labial folds temporally. Tear trough and malar groove depressions are marked, as well as areas of jowl fullness, submental fullness, and platysmal banding. The temporal browmidface incision is marked in a coronal orientation in the temporal scalp $\sim 2 \mathrm{~cm}$ into the hairline and $4 \mathrm{~cm}$ in length. An additional $3-\mathrm{cm}$ midline incision is marked in the hairline of patients who need corrugator release or frontalis scoring. Lower face and neck incisions follow a retro-tragal path around the ear and are extended in the postauricular scalp to provide access to the neck. Submental incisions are marked in the submental crease when needed.

\section{Technical Steps}

- Temporal brow incision.

- Endoscopic deep temporal fascial dissection: Sentinel vein identification and bisection of the temporal fascia.

- Zygo-orbicular ligament and malar retaining ligament release.

- Midface elevation and suture fixation.

- Brow dissection in supraperiosteal plane with selective superior brow retaining ligament release, corrugator cauterization, and galeal scoring.

- Brow fixation with galeal cable suture, dermal repositioning, and temporal flap fixation.

- Micro-fat grafting.

- Selective lower eyelid procedure.

\section{MIDFACE DISSECTION}

Access to the midface is obtained by extending the brow dissection over the superficial temporalis muscle fascia, deep to the temporoparietal and innominate fascia. At the level of the sentinel vein, the superficial temporal muscle fascia is scored so as to bifurcate the fascia establishing a plane that is followed down to the zygomatic arch. A medial to lateral dissection extending from the lateral rim out across the temporalis fascia is employed. Dissection follows the lateral rim and superior margin of the zygomatic arch. At the junction of the lateral one-third and medial two-thirds of the arch, a $1-\mathrm{cm}$ subperiosteal tunnel is developed over the arch. The periosteum is incised laterally, and then dissection is extended medially over the arch above the periosteum until the infraorbital rim is encountered. The reason for elevating the small subperiosteal tunnel is to create a point of anchorage along the course of the temporal branch of the facial nerve so as to avoid traction injury on the nerve when the midface is mobilized vertically. Dissection along the infraorbital rim continues in the sub-orbicularis oculi fat (SOOF) beneath the orbicularis oculi muscle. The zygo-orbicular ligament, which connects the preseptal orbicularis muscle to the infraorbital rim, is visualized and released (Fig. 6). This release spans 8 to $10 \mathrm{~mm}$ and completely liberates the orbicularis at the lid cheek junction. If excess orbital lid fat is present or if the orbital septum is lax, a transconjunctival preseptal dissection is performed. This connects the plane established by the midface dissection. The mobilized midface facilitates exposure to the fat compartments and orbital septum allowing fat removal and/or septal reset when required. The dissection then extends above the zygomaticus major muscle passing through the malar retaining ligament. Vertical spreading with tenotomy scissors is used to break through these strong connective tissue attachments and to spare the branches to the lateral orbicularis oculi muscle arising vertically 


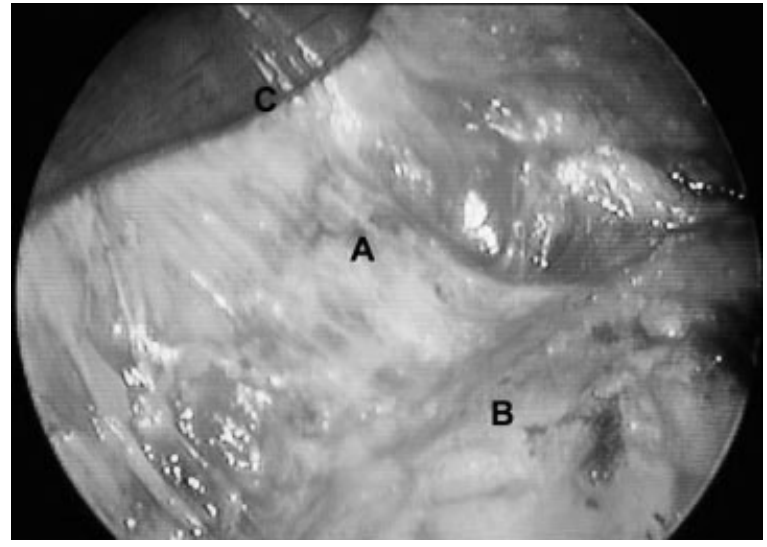

Figure 6 Endoscopic view of the zygo-orbicular ligament (lower eyelid retaining ligament): A, zygo-orbicular ligament; $B$, sub-orbicularis oculi fat (SOOF); C, preorbital orbicularis oculi.

from the zygomatic branch of the facial nerve (Fig. 7). The majority of these nerve branches are seen immediately lateral to the zygomatic major muscle. When the malar retaining ligament has been completely released, significant mobility of the midface is achieved (Fig. 8). Structures moved are the lateral portion of the orbicularis oculi muscle, the malar fat pad, and the high SMAS. To fix these structures, a single suture of 4-0 clear nylon is passed through the cephalic portion of the malar retaining ligament and anchored to the superficial temporal fascia just lateral to the lateral orbital rim (Figs. 9 and 10). This single suture is all that is required in the majority of patients. Additional orbicularis oculi sutures are used to shape the lower lid when required. When scleral show or lid laxity is present, a 5-0 clear nylon is placed in the pretarsal muscle and fixed to the deep head of the lateral canthal tendon. In the presence of a proptotic globe or negative vector orbit, a suture is

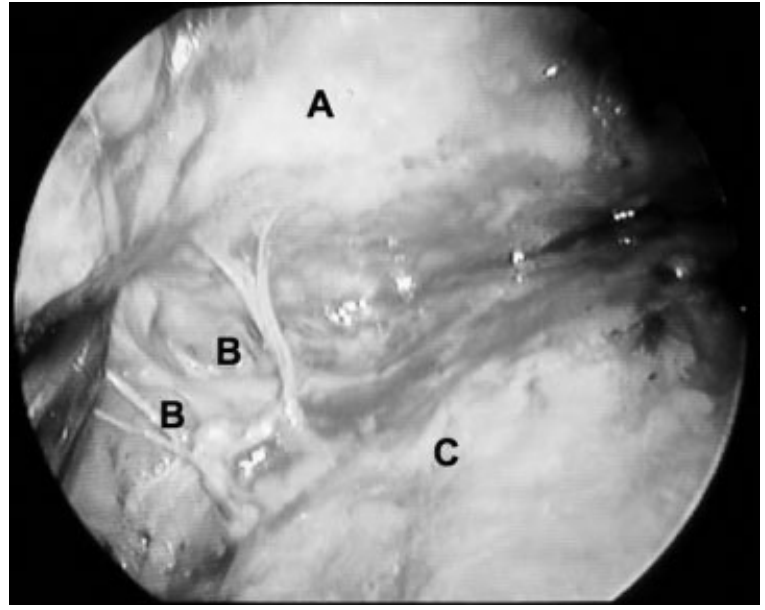

Figure 7 Endoscopic view of zygomatic nerve branches to orbicularis oculi: A, preorbital orbicularis oculi; B, zygomatic nerve branches to orbicularis oculi; $\mathrm{C}$, sub-orbicularis oculi fat (SOOF).

placed in the orbital portion of the orbicularis muscle and sutured directly cephalad along the infraorbital rim. This vertical movement of the orbicularis elevates the lid and eliminates "clothes lining" of the lid that can occur with a traditional canthopexy. The final suture variation involves direct release of the deep head of the lateral canthal tendon and repositioning cephalad. This maneuver is reserved for patients with true canthal malposition and has only been required in 3\% of patients. Lower eyelid skin is only removed if significant bunching is created with an approximate 2- to 3-mm excess. A croton oil peel may be applied for minor lower eyelid skin excess or crepy skin. Commonly, micro-fat grafting is performed in the malar groove, nasolabial folds, and lateral malar depression to blend any hollowing created by the vertical lift of the midface. The fat is harvested

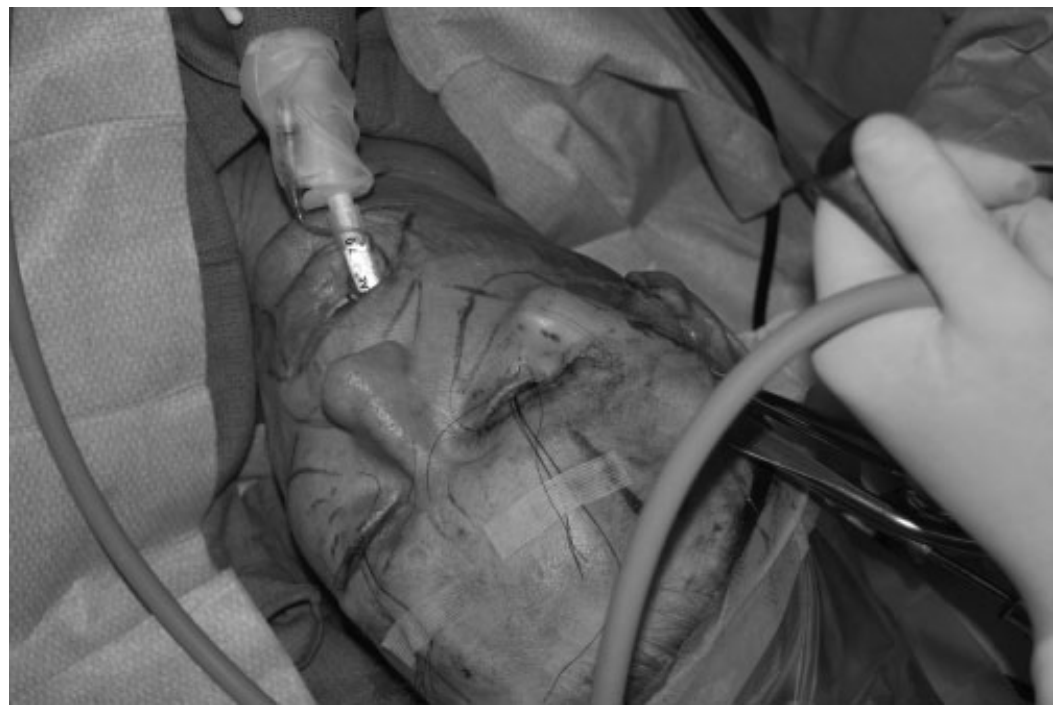

Figure 8 Intraoperative view of midface lift exposure with retractor elevation. 


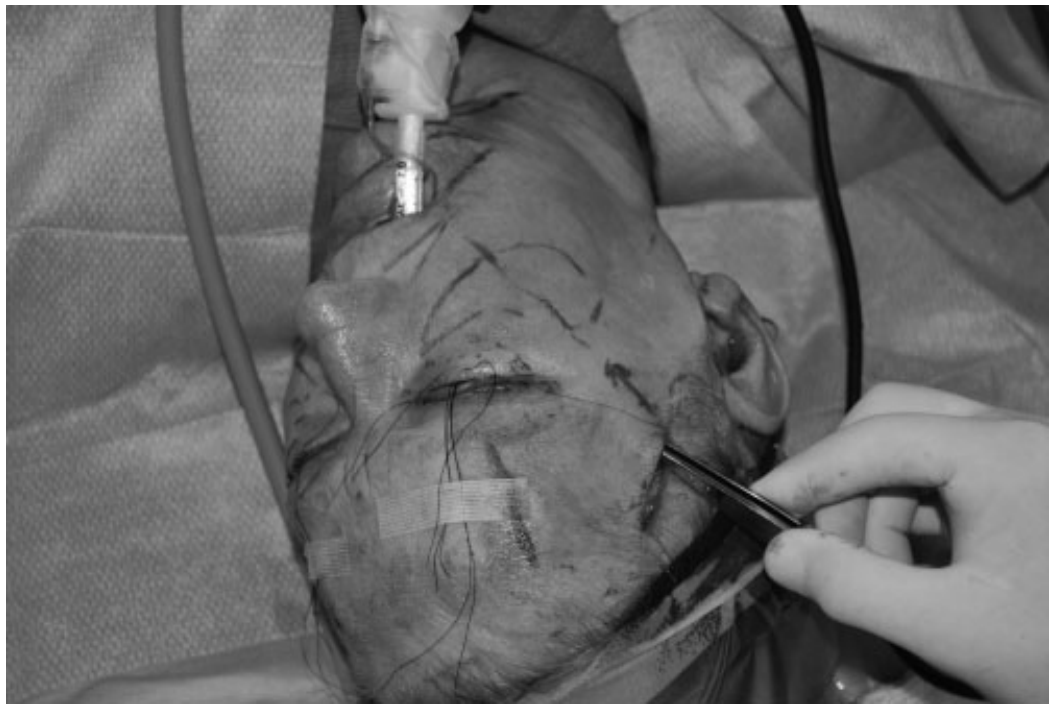

Figure 9 Intraoperative view of midface without point 1 elevation.

from an abdominal source at the beginning of the procedure and prepared for injection during the midface and brow dissection. Approximately 0.5 to $1 \mathrm{cc}$ of fat is injected into the malar groove, 2 to $3 \mathrm{cc}$ into the lateral malar area, and $2 \mathrm{cc}$ into the nasolabial folds. A small 7-mm perforated round drain is placed in the temporal incision and extends into the malar deadspace.

Two case examples are included with preoperative and postoperative results (Figs. 11 and 12).

\section{Criticisms and Downsides}

The main criticism of this procedure is the complexity of the anatomy as it is seen through the endoscope. The three-dimensional view of the sub-SMAS midface plane and the tactile sense of the tissues is lost with this approach. One must rely on anatomic relationships and bony landmarks to maintain a spatial sense during the dissection. The senior author has customized his instruments to allow for manipulation and use within the small operative field. One must have advanced endoscopic experience to be facile with the dissection and fixation of the midface and brow. As well, some would argue the long-term power within an endoscopic brow lift, though the senior author believes that with directed release of the brow and its retaining ligaments and cable suture fixation, long-term brow elevation can be achieved.

\section{POSTOPERATIVE CARE}

When the endoscopic brow midface procedure is done as a stand-alone procedure, an elastic band is placed around the forehead providing light compression and a way to

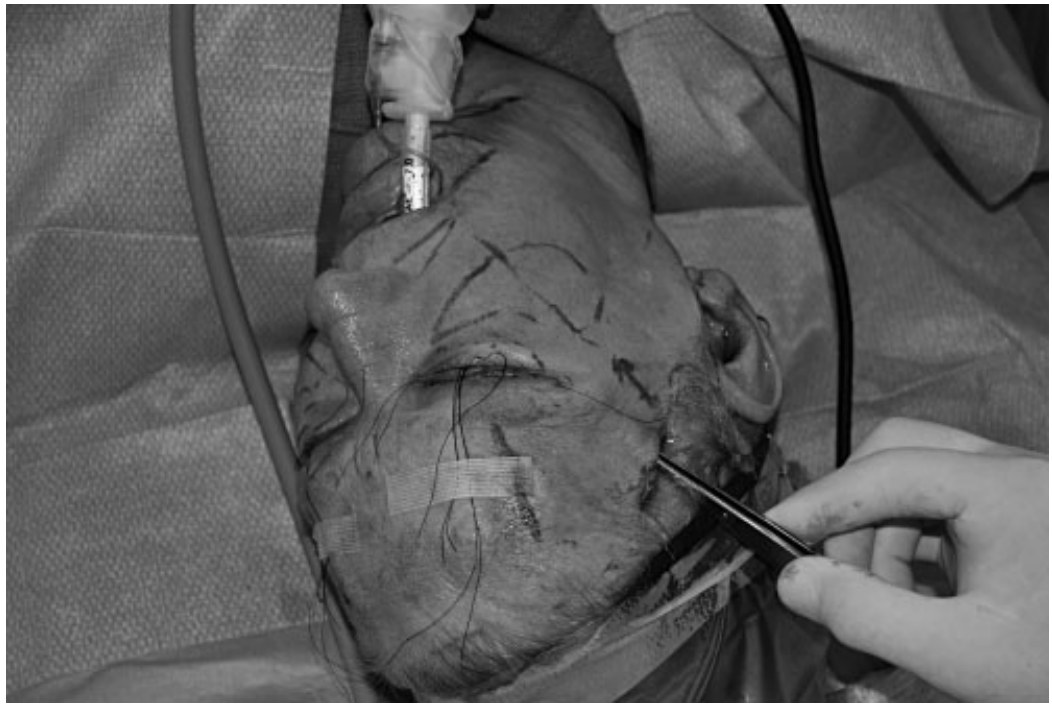

Figure 10 Intraoperative view of the midface with point 1 elevation. 

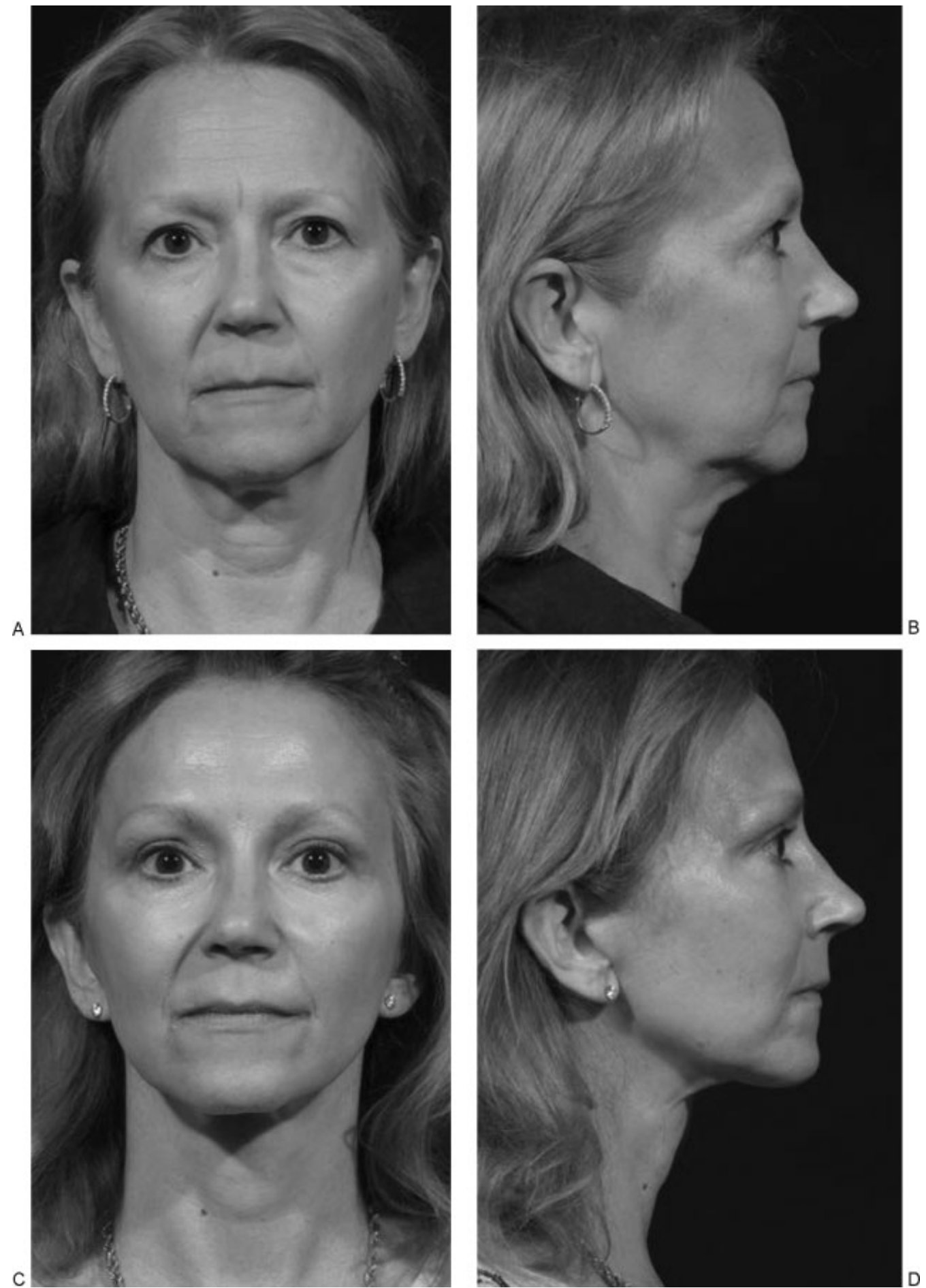

Figure 11 (A, B) Preoperative photographs of patient 1, a 49-year-old woman demonstrating the typical upper and mid face changes including lateral brow descent with deep forehead rhytides, upper lid fullness, malar fat pad descent, deep tear trough and malar groove, and an accentuated lid/cheek junction. The lower face and neck demonstrate soft tissue descent with jowling along the mandibular border and platysmal banding. (C, D) Postoperative photographs of patient 1, who underwent an endoscopic midface and brow lift, bilateral upper eyelid blepharoplasty, bilateral lower eyelid blepharoplasty with transconjunctival septal reset, and low SMAS neck lift with submental platysmal plication. The photographs demonstrate correction and rejuvenation of the aging brow, midface, and neck. 

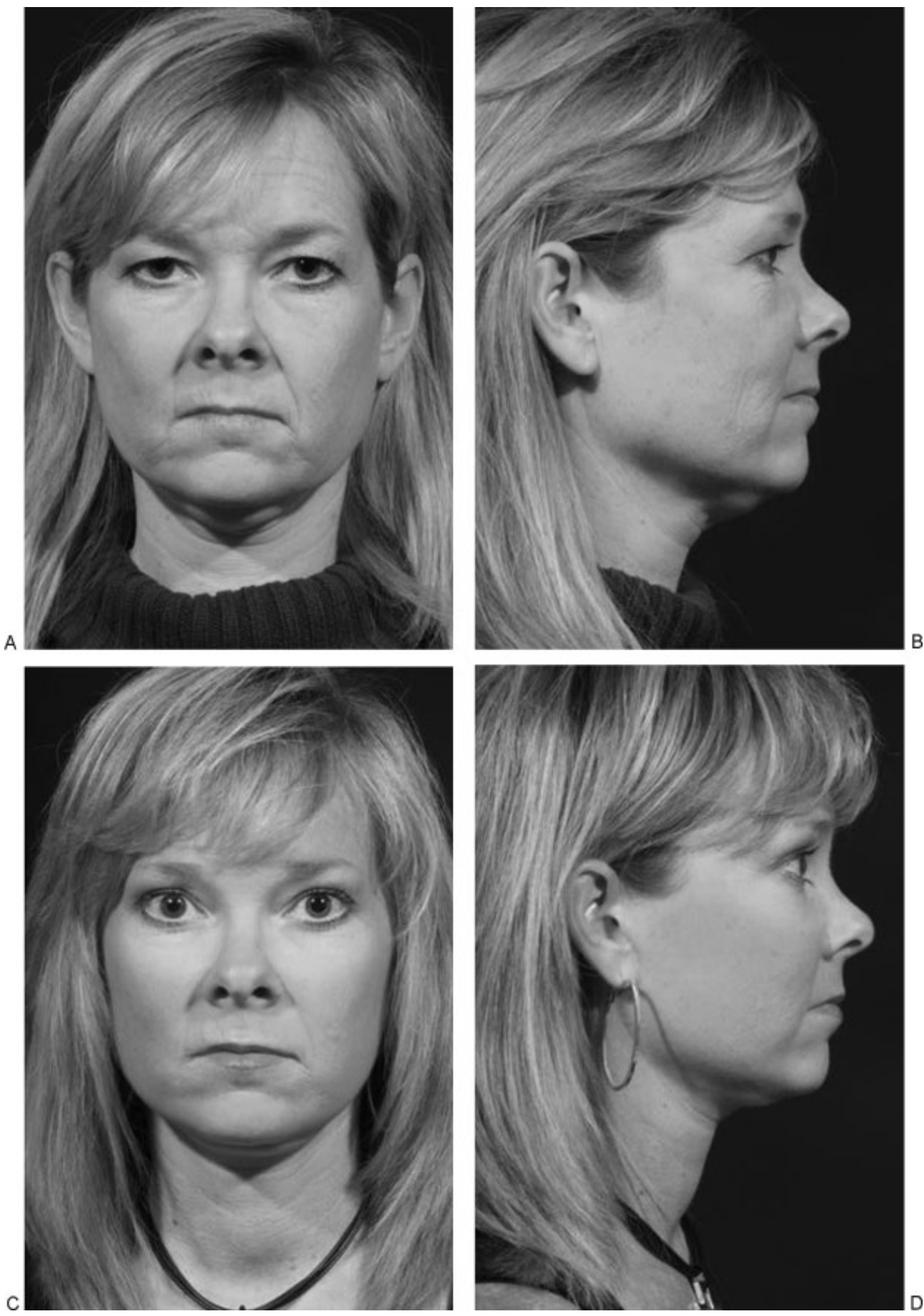

Figure 12 (A, B) Preoperative photographs of patient 2, a 39-year-old woman demonstrating the premature aging of the upper and mid face, including significant lateral brow descent with deep forehead rhytides, upper lid fullness, malar fat pad descent, a widened malar-orbicular crescent, deep tear trough and malar groove, and an accentuated lid/cheek junction. The lower face and neck demonstrate soft tissue descent with jowling along the mandibular border, significant perioral rhytides with volume loss, and platysmal banding. (C, D) Postoperative photographs of patient 2, who underwent an endoscopic midface and brow lift with galeal scoring, upper eyelid blepharoplasty, low SMAS neck lift with submental platysmal plication, and micro-fat injections to the lips. There is elevation of the brow with periorbital rejuvenation and blending of the lower eyelid-cheek junction. The midface soft tissue has been repositioned, the perioral volume augmented, and the neck contour improved. 
hold the closed suction drains that were placed through the temporal incision. When the procedure is combined with a low SMAS neck procedure, a conventional face-lift dressing that pads the neck and cheek area is employed. All patients receive $8 \mathrm{mg}$ Decadron IV (Dexamethasone, Abraxis Pharmaceutical Products, Schaumburg, IL) at the initiation of the procedure and are placed on a tapering dose pack that extends out to 5 days. All patients receive prophylactic antibiotics for 3 to 5 days. Drains are left in for 12 to 24 hours. A lighter dressing compressing the neck/jawline is reapplied. The dressing is removed at the time of suture removal on day 5 to 7 . An elastic neckband is worn at night for the next 5 days or until bruising has resolved. Makeup generally begins on the 10th day. Most patients return to work in 2 weeks but are advised that they will have tightness and excess lateral canthal tension out to 3 weeks postoperatively.

If patients undergo upper and/or lower blepharoplasty procedures, Swiss eye masks and aggressive eye lubrication are applied perioperatively for corneal protection. Tobradex eye drops (Tobramycin-dexamethasone, Alcon Laboratories Inc., Fort Worth, TX) are used for the septal reset patients.

\section{COMPLICATIONS}

The most common complication with the endoscopic midface procedure is asymmetry between the sides. Because fixation and position is suture dependent for the first 3 weeks after the procedure, we do not engage in massage to loosen or adjust the procedure until that time. Because no scalp has been removed, the site that is too tight can generally be loosened with topical massage over the points of suture fixation. It is important to point out asymmetries in brow and eyelid position prior to the surgery, as these will certainly be noted afterwards. No promise is made to correct extreme degrees of asymmetry, as overtightening the muscle on one side can produce expression asymmetries that are more notable than the passive asymmetry. Acute suture failure with loss of brow fixation occurred in $3 \%$ of 100 consecutive patients. This required immediate replacement of the suture. Loss of midface fixation has only been seen in a single elderly patient. Lower lid retraction, ectropion, and canthal malposition have been corrected rather than created by the midface procedure in conjunction with the orbicularis sutures described here. No occurrence of hematoma has been associated with the midface procedure. Nerve injury or skin loss has not occurred.

\section{REFERENCES}

1. Byrd HS, Andochick SE. The deep temporal lift: a multiplanar, lateral brow, temporal, and upper face lift. Plast Reconstr Surg 1996;97:928-937

2. Byrd HS, Salomon J. Endoscopic midface rejuvenation. In: Culbertson JH, ed. Operative Techniques in Plastic Surgery. Philadelphia, PA: WB Saunders; 1998:138-144

3. Byrd HS, Burt JD. Achieving aesthetic balance in the brow, eyelids, and midface. Plast Reconstr Surg 2002;110:926-933; discussion 934-939

4. Sullivan PK, Salomon JA, Woo AS, Freeman MB. The importance of the retaining ligamentous attachments of the forehead for selective eyebrow reshaping and forehead rejuvenation. Plast Reconstr Surg 2006;117:95-104

5. Mendelson BC, Muzaffar AR, Adams WP Jr. Surgical anatomy of the midcheek and malar mounds. Plast Reconstr Surg 2002;110:885-896; discussion 897-911

6. Lowe JB III, Cohen M, Hunter DA, Mackinnon SE. Analysis of the nerve branches to the orbicularis oculi muscle of the lower eyelid in fresh cadavers. Plast Reconstr Surg 2005;116: 1743-1749; discussion 1750-1751

7. Hamra ST. Frequent face lift sequelae: hollow eyes and the lateral sweep: cause and repair. Plast Reconstr Surg 1998;102: 1658-1666 